\title{
On groups, slow heat kernel decay yields Liouville property and sharp entropy bounds
}

\author{
Yuval Peres and Tianyi Zheng
}

\begin{abstract}
Let $\mu$ be a symmetric probability measure of finite entropy on a group $G$. We show that if $-\log \mu^{(2 n)}(\mathrm{id})=o\left(n^{1 / 2}\right)$, then the pair $(G, \mu)$ has the Liouville property (all bounded $\mu$-harmonic functions on $G$ are constant). Furthermore, if $-\log \mu^{(2 n)}$ (id) $=O\left(n^{\beta}\right)$ where $\beta \in(0,1 / 2)$, then the entropy of the $n$-fold convolution power $\mu^{(n)}$ satisfies $H\left(\mu^{(n)}\right)=O\left(n^{\frac{\beta}{1-\beta}}\right)$. These results improve earlier work of Gournay Gou16], Saloff-Coste and the second author [SCZ16]. We illustrate the sharpness of the bounds on a family of groups.
\end{abstract}

\section{Introduction}

Let $G$ be a finitely generated infinite group equipped with a generating set $S$, and let $\mu$ be a probability measure on $G$. Denote by $|g|$ the word distance of $g$ from the identity on the Cayley graph induced by $S$. Let $X_{1}, X_{2}, \ldots$ be a sequence of i.i.d. random variables with distribution $\mu$, so $W_{n}=X_{1} \cdots X$ is the random walk on $G$ with step distribution $\mu$. The law of $W_{n}$ is the $n$-fold convolution power $\mu^{(n)}$. The return probability of the $\mu$-random walk to the identity id after $2 n$ steps is

$$
\mathbf{P}\left(W_{2 n}=\mathrm{id}\right)=\mu^{(2 n)}(\mathrm{id}) .
$$

The Shannon entropy of $W_{n}$ is

$$
H_{\mu}(n)=H\left(W_{n}\right)=-\sum_{x \in G} \mu^{(n)}(x) \log \mu^{(n)}(x) .
$$

The rate of escape of $W_{n}$ is

$$
L_{\mu}(n)=\mathbf{E}\left|X_{n}\right|=\sum_{x \in G}|x| \mu^{(n)}(x)
$$

The pair $(G, \mu)$ has the Liouville property if all bounded $\mu$-harmonic functions on $G$ are constant. By classical work of Avez [Ave76], Derrienic [Der80 and KaimanovichVershik [KV83], for $\mu$ with finite entropy $H_{\mu}(1)<\infty$, the pair $(G, \mu)$ has the Liouville 
property if and only if the Avez asymptotic entropy $h_{\mu}=\lim _{n \rightarrow \infty} \frac{H_{\mu}(n)}{n}$ is 0 . We say a probability measure $\mu$ on $G$ is symmetric if $\mu(g)=\mu\left(g^{-1}\right)$ for all $g \in G$.

Our goal in this article is to show a link between the decay of the return probability and the growth of entropy of a symmetric random walk on $G$. Namely, we derive an upper bound on $H_{\mu}(n)$ from a lower bound on $\mu^{(2 n)}(\mathrm{id})$, provided that $\mu^{(2 n)}(\mathrm{id})$ decays sufficiently slowly.

Theorem 1.1. Suppose $\mu$ is a symmetric probability measure of finite entropy on $G$ such that

$$
\mu^{(2 n)}(\mathrm{id}) \geq \exp (-\gamma(n))
$$

where $\gamma:[1, \infty) \rightarrow \mathbb{R}_{+}$is an unbounded function such that both $\gamma(n)$ and $n^{\frac{1}{2}} / \gamma(n)$ are increasing, that satisfies

$$
\lim _{n \rightarrow \infty} \frac{\gamma(n)}{n^{\frac{1}{2}}}=0
$$

Then $(G, \mu)$ has the Liouville property.

The decay of the return probability enjoys good stability properties, in particular the asymptotic decay of return probability of simple random walk is a quasi-isometry invariant, see Pittet and Saloff-Coste [PSC00]. However, it remains a major open problem whether the Liouville property is stable under changing the generating set of the group. We deduce from Theorem 1.1 the following corollary regarding stability of the Liouville property provided that $\mu^{(2 n)}(\mathrm{id})$ decays slower than $\exp \left(-n^{1 / 2}\right)$. We say a probability measure $\mu$ on $G$ has finite second moment if $\sum_{g \in G}|g|^{2} \mu(g)<\infty$.

Corollary 1.2. Suppose $G$ is a finitely generated group such that for some symmetric probability measure $\mu$ with finite generating support on $G, \mu^{(2 n)}$ (id) satisfies the assumptions in Theorem 1.1. Let $\Gamma$ be a finitely generated group that is quasiisometric to $G$. Then $(\Gamma, \eta)$ has the Liouville property for any symmetric probability measure $\eta$ of finite second moment on $\Gamma$.

Simple random walk on the lamplighter group over the two-dimensional lattice $G=\mathbb{Z}_{2} 2 \mathbb{Z}^{2}$ satisfies $\mu^{(2 n)}(\mathrm{id}) \simeq \exp \left(-n^{1 / 2}\right)$ and $H_{\mu}(n) \simeq n / \log n$, see Ers03, Ers06, PSC02]. This example is just beyond the limit of application of Theorem 1.1. Kotowski and Virág [KV15] analyzed a group $G$ on which simple random walk satisfies $\mu^{(2 n)}(\mathrm{id}) \gtrsim \exp \left(-n^{1 / 2+o(1)}\right)$ and the entropy $H_{\mu}(n)$ has linear growth. The Kotowski-Virág example shows that the exponent $1 / 2$ is the critical value in the setting of Theorem 1.1. It is an interesting open problem whether $\mu^{(2 n)}(\mathrm{id}) \gtrsim \exp \left(-n^{\frac{1}{2}}\right)$ implies that $(G, \mu)$ has the Liouville property.

By a result of Kaimanovich Kai91, if $\mu$ is a symmetric probability measure with finite first moment on a polycyclic group $G$, then $(G, \mu)$ has the Liouville

\footnotetext{
${ }^{1}$ Given two monotone functions $\phi, \psi$, write $\phi \gtrsim \psi$ if there are constants $c_{1}, c_{2} \in(0, \infty)$, such that $\phi(t) \geq c_{1} \psi\left(c_{2} t\right)$ (using integer values if $\phi, \psi$ are defined on $\mathbb{N}$ ). We write $\psi \simeq \phi$ if both $\phi \gtrsim \psi$ and $\psi \gtrsim \phi$ holds.
} 
property. By Alexopoulos Ale92, Coulhon-Grigor'yan-Pittet CGP01, Theorem 7.10], for simple random walk on a polycyclic group of exponential volume growth, the return probability satisfies $\mu^{(2 n)}(\mathrm{id}) \simeq \exp \left(-n^{1 / 3}\right)$. As a corollary of Theorem 1.1. we extend the aforementioned result of Kaimanovich to groups where the return probability of simple random walk decays no faster than $\exp \left(-n^{1 / 3}\right)$.

Corollary 1.3. Suppose $G$ is a finitely generated group such that for some symmetric probability measure $\mu$ with finite generating support on $G$,

$$
\mu^{(2 n)}(\mathrm{id}) \gtrsim \exp \left(-n^{1 / 3}\right) \text {. }
$$

Then $(G, \eta)$ has the Liouville property for every symmetric probability measure $\eta$ on $G$ with finite first moment $\sum_{g \in G}|g| \eta(g)<\infty$.

So far all known examples of groups that satisfy the assumption of Corollary 1.3 belong to the class of geometrically elementary solvable groups (GES), defined in Tessera [Tes13]. See Section 4 for the more details.

When the decay of the return probability $\mu^{(2 n)}(\mathrm{id})$ is much slower than $\exp \left(-n^{\frac{1}{2}}\right)$, we have the following explicit entropy upper bound. It improves a bound from SCZ16.

Theorem 1.4. Let $\mu$ be a symmetric probability measure of finite entropy on $G$. Suppose there exists constants $C>0, \beta \in\left(0, \frac{1}{2}\right)$ such that

$$
\mu^{(2 n)}(\mathrm{id}) \geq \exp \left(-C n^{\beta}\right) \text {. }
$$

Then there exists a constant $C_{1}=C_{1}(\beta, C)$ such that

$$
H_{\mu}(n) \leq C_{1} n^{\frac{\beta}{1-\beta}} .
$$

Remark 1.5. The bound stated above is a special case of Theorem 3.2 A larger class of lower bound functions on the return probability can be converted into entropy upper bounds. For example, suppose there exists constants $C>0, \beta \in\left(0, \frac{1}{2}\right)$ and $\kappa \in \mathbb{R}$ such that

$$
\mu^{(2 n)}(\mathrm{id}) \geq \exp \left(-C n^{\beta} \log ^{\kappa}(n+1)\right),
$$

then there exists a constant $C_{1}=C_{1}(\beta, \kappa, C)$ such that

$$
H_{\mu}(n) \leq C_{1} n^{\frac{\beta}{1-\beta}} \log ^{\frac{\kappa}{1-\beta}}(n+1) .
$$

More details can be found in Section 3 . 
Based on the spectral profile of balls in the group $G$, we derive an upper bound on the rate of escape $L_{\mu}(n)$. Given a symmetric probability measure $\mu$ on $G$ and a function $f \in \ell^{2}(G)$, consider the associated Dirichlet form

$$
\mathcal{E}_{\mu}(f)=\frac{1}{2} \sum_{x, y \in G}(f(x y)-f(x))^{2} \mu(y),
$$

and define

$$
\lambda_{\mu}(\Omega)=\inf \left\{\mathcal{E}_{\mu}(f): \operatorname{support}(f) \subset \Omega,\|f\|_{2}=1\right\} .
$$

In words, $\lambda_{\mu}(\Omega)$ is the lowest eigenvalue of the operator of convolution by $\delta_{e}-\mu$ with Dirichlet boundary condition in $\Omega$. This operator is associated with the discrete time Markov process corresponding to the $\mu$-random walk killed outside $\Omega$.

Theorem 1.6. Let $\mu$ be a symmetric probability measure on $G,\left(W_{n}\right)$ be a $\mu$-random walk on $G$. Suppose there exists constants $C>0$ and $\theta>0$ such that

$$
\lambda_{\mu}(B(\mathrm{id}, r)) \leq C r^{-\theta},
$$

then for any $\alpha \in(0, \theta)$, there exists a constant $C_{1}=C_{1}(C, \theta, \alpha)$ such that

$$
\mathbf{E} \max _{0 \leq k \leq n}\left|W_{k}\right|^{\alpha} \leq C_{1} n^{\alpha / \theta}
$$

The stability of the asymptotic behavior of the function $r \rightarrow \lambda_{\mu}(B(\mathrm{id}, r))$ was investigated by Tessera in [Tes13]. In particular, in [Tes13] it was proved that an upper bound of the form $\lambda_{\mu}(B(\mathrm{id}, r))=O(g(C r))$ when $r \rightarrow \infty$ for some monotone function $g$ is stable under quasi-isometry, taking finite products, quotients and finitely generated subgroups. From the stability results we deduce the following corollary.

Corollary 1.7. Let $G$ be a finitely generated group that is quasi-isometric to a polycyclic group. Then for any symmetric probability measure $\mu$ on $G$ with finite second moment, there exists a constant $C>0$ such that

$$
L_{\mu}(n) \leq \mathbf{E} \max _{0 \leq k \leq n}\left|W_{k}\right| \leq C n^{\frac{1}{2}} .
$$

Theorem 1.6 is a special case of Theorem 4.2, and Corollary 1.7 is a special case of Corollary 4.5. In Section 5 we illustrate the sharpness of Theorem 1.4.

Proposition 1.8. For any $\beta \in\left[\frac{1}{3}, \frac{1}{2}\right)$, there exists a group $G$ and a symmetric probability measure $\mu$ of generating finite support on $G$ such that

$$
\begin{aligned}
\mu^{(2 n)}(\mathrm{id}) & \simeq \exp \left(-n^{\beta} \log ^{1-\beta} n\right), \\
H_{\mu}(n) & \simeq n^{\frac{\beta}{1-\beta}} \log n .
\end{aligned}
$$

Theorem 1.1, 1.4 and 1.6 extend to symmetric random walks on transitive graphs. In Section 6, we explain how the results carry over. 


\section{Related works}

The idea of connecting slow decay of the return probability to the Liouville property was first proposed by Gournay in Gou16. The Hilbert compression exponent $\alpha_{2}^{*}(G)$ was introduced in Guentner and Kaminker [GK04, who defined $\alpha_{2}^{*}(G)$ as the supremum over all $\alpha \geq 0$ such that there exists a 1-Lipschitz map $f: G \rightarrow \mathcal{H}$ and a constant $c>0$ such that $\|f(x)-f(y)\|_{\mathcal{H}} \geq c d_{G}(x, y)^{\alpha}$. Analogously, one can consider the equivariant Hilbert compression exponent $\alpha_{2}^{\#}(G)$, which is defined in the same way as $\alpha_{2}^{*}(G)$ with the additional requirement that $f$ is equivariant (see Section 3 for the definition). It is known that for finitely generated amenable groups, $\alpha_{2}^{*}(G)=\alpha_{2}^{\#}(G)$, see dCTV07. One result from Gou16] is the following. Suppose $\mu$ is a symmetric probability measure of finite support on $G$ such that $\mu^{(2 n)}(\mathrm{id}) \geq C_{1} \exp \left(-C_{2} n^{\gamma}\right)$ for some $\gamma \in(0,1)$, then Gou16, Theorem 1.1]

$$
\alpha_{2}^{\#}(G) \geq \frac{1-\gamma}{1+\gamma}
$$

Let $\beta^{*}(\mu)$ be the upper speed exponent, $\beta^{*}(\mu):=\lim _{\sup } \rightarrow \infty \frac{\log L_{\mu}(n)}{\log n}$. From Austin, Naor and Peres [ANP09, Proposition 1.1],

$$
\alpha_{2}^{\#}(G) \leq \frac{1}{2 \beta^{*}(\mu)}
$$

Therefore Gournay's result implies that when $\mu^{(2 n)}(\mathrm{id}) \geq C_{1} \exp \left(-C_{2} n^{\gamma}\right)$, we have

$$
\beta^{*}(\mu) \leq \frac{1+\gamma}{2(1-\gamma)}
$$

By a result of Varopoulos, we have $\mu^{(2 n)}(i d) \lesssim \exp \left(-n^{\frac{1}{3}}\right)$ for simple random walks on groups of exponential volume growth. The upper bound (3) on $\beta^{*}(\mu)$ is not strong enough to imply the Liouville property of $(G, \mu)$ when $\gamma \in[1 / 3,1 / 2)$.

In the work of the second author with Saloff-Coste [SCZ16], an upper bound similar to Theorem 1.4 was proved. More precisely, suppose $\mu$ is a symmetric probability measure of finite second moment on $G$ such that $\mu^{(2 n)}(\mathrm{id}) \geq C_{1} \exp \left(-C_{2} n^{\gamma}\right)$ for some $\gamma \in(0,1 / 2)$, then by [SCZ16, Theorem 1.7]

$$
H_{\mu}(n) \lesssim\left(n \log ^{1+\epsilon} n\right)^{\frac{\gamma}{1-\gamma}} \text { for any } \epsilon>0 .
$$

In particular, this bound implies the Liouville property of $(G, \mu)$ for $\gamma \in(0,1 / 2)$. Our results in this paper imply a sharper upper bound $H_{\mu}(n) \lesssim n^{\frac{\gamma}{1-\gamma}}$ under the same assumption, and are applicable to a larger class of decay lower bounds.

The following bound on the upper speed exponent is known through work of Tessera [Tes11], Austin, Naor and Peres ANP09. Suppose there exists constants $C>0$ and $\theta>0$ such that

$$
\lambda_{\mu}(B(\mathrm{id}, r)) \leq C r^{-\theta},
$$


then

$$
\beta^{*}(\mu):=\limsup _{n \rightarrow \infty} \frac{\log L_{\mu}(n)}{\log n} \leq \frac{1}{\theta} .
$$

This relation is proved via the equivariant Hilbert compression exponent $\alpha_{2}^{\#}(G)$. Indeed, by [Tes11, Theorem 10], the assumption $\lambda_{\mu}(B(\mathrm{id}, r)) \leq C r^{-\theta}$ implies that

$$
\alpha_{2}^{\#}(G) \geq \frac{\theta}{2}
$$

By [ANP09, Proposition 1.1], we have

$$
\beta^{*}(\mu) \leq \frac{1}{2 \alpha_{2}^{\#}(G)} \leq \frac{1}{\theta} .
$$

Theorem 1.6 provides a more precise upper bound on $L_{\mu}(n)$ than just the upper speed exponent.

\section{The spectral profile}

The spectral profile $\Lambda_{G, \mu}$ of a symmetric probability measure $\mu$ on $G$ is defined as

$$
\Lambda_{G, \mu}(v)=\Lambda_{\mu}(v)=\inf \left\{\lambda_{\mu}(\Omega): \Omega \subset G,|\Omega| \leq v\right\},
$$

where $\lambda_{\mu}(\Omega)$ is the lowest Dirichlet eigenvalue defined in (11). We now review work of Coulhon [Cou96] which relates the behavior of $n \mapsto \mu^{(2 n)}$ (id) to the spectral profile $v \mapsto \Lambda_{\mu}(v)$. See Cou96] for references to earlier related works, in particular, work of Grigor'yan in which the spectral profile plays a key role. By Cou96, Proposition II.1], we have

$$
\mu^{(2 n+2)}(\mathrm{id}) \leq 2 \psi(2 n),
$$

where $\psi:[0,+\infty) \rightarrow[1,+\infty)$ is defined implicitly by

$$
t=\int_{1}^{1 / \psi(t)} \frac{d s}{2 s \Lambda_{\mu}(4 s)} .
$$

From Coulhon's result one can deduce the following useful lemma, see SCZ16, Lemma 2.5]. We include a proof here for the reader's convenience.

Lemma 2.1. Assume that $\mu^{(2 n)}(\mathrm{id}) \geq \exp (-\gamma(n))$ where $\gamma:[1, \infty) \rightarrow(0, \infty)$ is an increasing function and $\lim _{n \rightarrow \infty} \gamma(n)=\infty$. Then for all $n \in \mathbb{N}$ we have

$$
\Lambda_{\mu}\left(8 e^{\gamma(n)}\right) \leq \frac{\gamma(n)+\log 8}{2 n}
$$


Proof. Let $\psi$ be defined in terms of $\Lambda_{\mu}$ as in (6). By definition of $\psi$ and the fact that $\Lambda_{\mu}$ is a non-increasing function, we have

$$
t=\int_{1}^{1 / \psi(t)} \frac{d s}{2 s \Lambda_{\mu}(4 s)} \leq \frac{\log (4 / \psi(t))}{2 \Lambda_{\mu}(4 / \psi(t))}
$$

which we rewrite as

$$
\Lambda_{\mu}(4 / \psi(t)) \leq \frac{\log (4 / \psi(t))}{2 t}
$$

By [Cou96, Proposition II.1] and the hypothesis,

$$
e^{-\gamma(n+1)} \leq \mu^{(2 n+2)}(\mathrm{id}) \leq 2 \psi(2 n)
$$

Hence

$$
\Lambda_{\mu}\left(8 e^{\gamma(n+1)}\right) \leq \Lambda_{\mu}(4 / \psi(2 n)) \leq \frac{\log (4 / \psi(2 n))}{4 n} \leq \frac{\gamma(n+1)+\log 8}{4 n} .
$$

\section{Upper bounds on entropy}

In this section we prove Theorem 1.1, 1.4 and Corollary 1.2, We will use 1-coboundaries constructed from test functions provided by the spectral profile, together with the Markov type inequality to bound the probability that the random walk deviates from its typical behavior. Throughout this section, let $\left(W_{n}\right)$ be a $\mu$-random walk on $G$, where $\mu$ is a symmetric probability measure of finite entropy $H_{\mu}(1)<\infty$.

Recall that $f: G \rightarrow \mathcal{H}$, where $\mathcal{H}$ is a Hilbert space, is called a 1-cocycle if there exists a representation $\pi: G \rightarrow \operatorname{Isom}(\mathcal{H})$ such that $f(g x)=\pi_{g} f(x)+f(g)$. The space of 1-cocycles associated with representation $\pi$ is denoted by $Z^{1}(G, \pi)$. The space of 1-coboundaries, $B^{1}(G, \pi)=\left\{f: G \rightarrow \mathcal{H}: \exists v \in \mathcal{H}, f(g)=\pi_{g} v-v\right\}$, is a subspace of $Z^{1}(G, \pi)$. We say a map $f: G \rightarrow \mathcal{H}$ is equivariant if $f \in Z^{1}(G, \pi)$ for some representation $\pi: G \rightarrow \operatorname{Isom}(\mathcal{H})$.

The following Markov type inequality is a special case of [NP08, Theorem 2.1], see also [NP08, Remark 2.6]: for any $f \in Z^{1}(G, \pi)$, we have for all $n \in \mathbb{N}$,

$$
\mathbf{E}\left[\left\|f\left(W_{n}\right)\right\|_{\mathcal{H}}^{2}\right] \leq n \mathbf{E}\left[\left\|f\left(W_{1}\right)\right\|_{\mathcal{H}}^{2}\right] .
$$

Note that if we choose $f$ to be a 1-coboundary, $f(g)=\pi_{g} v-v$, then $\|f(g)\|_{\mathcal{H}} \leq$ $2\|v\|_{\mathcal{H}}$ is bounded. In particular, if we apply (7) to 1-coboundaries, quantities on both sides are finite. The following lemma holds for all symmetric random walks on $G$.

Lemma 3.1. Let $\mu$ be a symmetric probability measure on $G$, and $U$ be a finite subset of $G$. Then for a $\mu$-random walk $\left(W_{n}\right)$ on $G$,

$$
\mathbf{P}\left(W_{n} \notin U^{-1} U\right) \leq n \lambda_{\mu}(U) .
$$


Proof. By the definition of $\lambda_{\mu}(U)$, for any $\epsilon>0$, there exists a function $\phi$ supported on $U$ such that

$$
\frac{\mathcal{E}_{\mu}(\phi)}{\|\phi\|_{2}^{2}} \leq \lambda_{\mu}(U)+\epsilon
$$

Consider the 1-coboundary

$$
\begin{aligned}
\sigma: G & \rightarrow \ell^{2}(G) \\
\sigma(g) & =\tau_{g} \phi-\phi,
\end{aligned}
$$

where $\tau_{g}$ is the right translation $\tau_{g} \phi(x)=\phi(x g)$. By the Markov type inequality in Hilbert space (7), we have

$$
\mathbf{E}\left[\left\|\sigma\left(W_{n}\right)\right\|_{2}^{2}\right] \leq n \mathbf{E}\left[\left\|\sigma\left(W_{1}\right)\right\|_{2}^{2}\right] .
$$

Note that

$$
\mathbf{E}\left[\left\|\sigma\left(W_{1}\right)\right\|_{2}^{2}\right]=2 \mathcal{E}_{\mu}(\phi) .
$$

On the other hand, if $g \notin U^{-1} U$, then supp $\phi \cap \operatorname{supp} \tau_{g} \phi=\emptyset$, and it follows that

$$
\|\sigma(g)\|_{2}^{2}=\|\phi\|_{2}^{2}+\left\|\tau_{g} \phi\right\|_{2}^{2}=2\|\phi\|_{2}^{2} .
$$

Therefore

$$
2\|\phi\|_{2}^{2} \mathbf{P}\left(W_{n} \notin U^{-1} U\right) \leq \mathbf{E}\left[\left\|\sigma\left(W_{n}\right)\right\|_{2}^{2}\right] \leq 2 n \mathcal{E}_{\mu}(\phi) .
$$

From the choice of function $\phi$, we have

$$
\mathbf{P}\left(W_{n} \notin U^{-1} U\right) \leq n \frac{\mathcal{E}_{\mu}(\phi)}{\|\phi\|_{2}^{2}} \leq n\left(\lambda_{\mu}(U)+\epsilon\right) .
$$

Since $\epsilon>0$ is an arbitrary positive number, we obtain the statement.

To proceed, for each $k \in \mathbb{N}$, let $U_{k}$ be a set of size $\left|U_{k}\right|=\left\lceil e^{e^{k}}\right\rceil$ such that

$$
\lambda_{\mu}\left(U_{k}\right) \leq 2 \Lambda_{\mu}\left(e^{e^{k}}\right)
$$

By Lemma 2.1, the assumption $\mu^{(2 n)}(\mathrm{id}) \geq \exp (-\gamma(n))$ implies

$$
\Lambda_{\mu}\left(e^{e^{k}}\right) \leq \frac{e^{k}}{2 \gamma^{-1}\left(e^{k}-\log 8\right)} \leq \frac{1}{2 e^{k} \varphi\left(e^{k} / 2\right)} \text { for all } k \geq k_{0}=\log (\gamma(2)+\log 64),
$$

where $\varphi(x)=\frac{\gamma^{-1}(x)}{x^{2}}$. Note that if $x^{-\frac{1}{2}} \gamma(x)$ decreases to 0 as $x \rightarrow \infty$, then $\varphi(x)$ is increasing and $\lim _{x \rightarrow \infty} \varphi(x)=\infty$. 
Proof of Theorem [1.1. For a given time $n$, define

$$
\ell_{n}=\min \left\{k: e^{k} \varphi^{\frac{1}{2}}\left(e^{k} / 2\right) \geq n\right\} .
$$

For example, when $\gamma(n)=C n^{\beta}$ for some $\beta \in(0,1 / 2)$, then $\varphi(x)=C^{-1 / \beta} x^{1 / \beta-2}$ and $\ell_{n} \sim \log \left(n^{\frac{\beta}{1-\beta}}\right)$.

By Lemma 3.1, we have

$$
\mathbf{P}\left(W_{n} \notin U_{\ell_{n}}^{-1} U_{\ell_{n}}\right) \leq 2 n \Lambda_{\mu}\left(e^{e^{\ell_{n}}}\right) \leq \frac{2 n}{2 e^{\ell_{n}} \varphi\left(e^{\ell_{n}} / 2\right)} \leq \frac{1}{\varphi^{\frac{1}{2}}\left(e^{\ell_{n}}\right)} .
$$

This implies that

$$
\lim _{n \rightarrow \infty} \mathbf{P}\left(W_{n} \notin U_{\ell_{n}}^{-1} U_{\ell_{n}}\right)=0 .
$$

Assume now that the asymptotic entropy $h_{\mu}>0$. Then by Shannon theorem, see for example [LP16, Theorem 14.10], for any $\epsilon>0$, there exists $n_{\epsilon}$ such that for all $n \geq n_{\epsilon}$,

$$
\mathbf{P}\left(W_{n} \in U\right) \leq \epsilon+|U| e^{-n h_{\mu} / 2} .
$$

Note that since $e^{\ell_{n}-1} \varphi^{\frac{1}{2}}\left(e^{\ell_{n}-1} / 2\right)<n$, we have

$$
\log \left|U_{\ell_{n}}^{-1} U_{\ell_{n}}\right| \leq 2 \log \left|U_{\ell_{n}}\right|=2 e^{\ell_{n}}<\frac{2 e n}{\varphi^{\frac{1}{2}}\left(e^{\ell_{n}-1} / 2\right)} .
$$

Therefore if $h_{\mu}>0$, we have

$$
\lim _{n \rightarrow \infty} \mathbf{P}\left(W_{n} \in U_{\ell_{n}}^{-1} U_{\ell_{n}}\right)=0,
$$

which contradicts (11). We conclude that $h_{\mu}=0$.

We now derive Corollary 1.2 and 1.3 from Theorem 1.1 .

Proof of Corollary 1.2. By work of Pittet and Saloff-Coste [PSC00, Theorem 1.4], if $\mu_{i}, i=1,2$, are symmetric probability measures on $G$ with generating support and finite second moment $\sum_{g \in G}|g|^{2} \mu_{i}(g)<\infty$, then the functions $n \mapsto \mu_{i}^{(2 n)}$ (id) satisfy $\mu_{1}^{(2 n)}(\mathrm{id}) \simeq \mu_{2}^{(2 n)}(\mathrm{id})$. Further, the equivalence class of the decay function $\mu_{1}^{(2 n)}$ (id) is an invariant of quasi-isometry, see PSC00. Combine this stability result with Theorem 1.1, we obtain the statement.

Proof of Corollary 1.3. From the proof of Theorem 1.1, it is sufficient to show that under the assumptions of the statement, for any symmetric probability measure $\eta$ on $G$ with finite first moment, we have

$$
\Lambda_{\eta}\left(e^{x}\right) \leq \frac{1}{x \varphi(x)}, \text { where } \varphi(x) \rightarrow \infty \text { as } x \rightarrow \infty
$$


To obtain such an estimate, we use a truncation argument similar to the proof of [SCZ16, Theorem 2.13].

We may assume that $\mu$ is uniform on a symmetric finite generating set $S$ of $G$. By Lemma 2.1, the assumption that $\mu^{(2 n)}(\mathrm{id}) \gtrsim \exp \left(-n^{1 / 3}\right)$ implies that there is a constant $C>0$ such that for all $r \geq 1$,

$$
\Lambda_{\mu}\left(e^{r}\right) \leq \frac{C}{r^{2}} .
$$

Recall the pseudo-Poincaré inequality (see for example [PSC00])

$$
\left\|f-\tau_{g} f\right\|_{\ell^{2}(G)}^{2} \leq 2|S||g|^{2} \mathcal{E}_{\mu}(f),
$$

where $\tau_{g}$ is the right translation $\tau_{g} f(x)=f(x g)$. For any function $f$ with finite support, we have

$$
\begin{aligned}
\mathcal{E}_{\eta}(f) & =\frac{1}{2} \sum_{|g| \leq r}\left\|f-\tau_{g} f\right\|_{\ell^{2}(G)}^{2} \eta(g)+\frac{1}{2} \sum_{|g|>r}\left\|f-\tau_{g} f\right\|_{\ell^{2}(G)}^{2} \eta(g) \\
& \leq \frac{1}{2} \sum_{|g| \leq r}\left\|f-\tau_{g} f\right\|_{\ell^{2}(G)}^{2} \eta(g)+\|f\|_{\ell^{2}(G)}^{2} \eta\{g:|g| \geq r\} .
\end{aligned}
$$

By the pseudo-Poincaré inequality (12), we have

$$
\sum_{|g| \leq r}\left\|f-\tau_{g} f\right\|_{\ell^{2}(G)}^{2} \eta(g) \leq 2|S| \mathcal{E}_{\mu}(f) \sum_{|g| \leq r}|g|^{2} \eta(g) .
$$

Therefore

$$
\frac{\mathcal{E}_{\eta}(f)}{\|f\|_{\ell^{2}(G)}^{2}} \leq|S|\left(\sum_{|g| \leq r}|g|^{2} \eta(g)\right) \frac{\mathcal{E}_{\mu}(f)}{\|f\|_{\ell^{2}(G)}^{2}}+\eta(\{g:|g| \geq r\}) .
$$

Now we restrict to functions with $|\operatorname{supp} f| \leq e^{r}$, then it follows that

$$
\begin{aligned}
\Lambda_{\eta}\left(e^{r}\right) & \leq|S|\left(\sum_{|g| \leq r}|g|^{2} \eta(g)\right) \Lambda_{\mu}\left(e^{r}\right)+\eta(\{g:|g| \geq r\}) \\
& \leq C|S| r^{-2}\left(\sum_{|g| \leq r}|g|^{2} \eta(g)\right)+\eta(\{g:|g| \geq r\}) .
\end{aligned}
$$

Since $\eta$ has finite first moment, $\sum_{g \in G}|g| \eta(g)<\infty$, we have $\sum_{|g| \geq r}|g| \eta(g) \rightarrow 0$ as $r \rightarrow \infty$. Therefore, $r \eta(\{g:|g| \geq r\}) \rightarrow 0$ as $r \rightarrow \infty$, and

$$
\begin{aligned}
\frac{1}{r} \sum_{g:|g| \leq r}|g|^{2} \eta(g) & \leq \frac{1}{r}\left(r^{1 / 2} \sum_{|g| \leq r^{1 / 2}}|g| \eta(g)+r \sum_{|g| \geq r^{1 / 2}}|g| \eta(g)\right) \\
& \leq r^{-1 / 2} \sum_{g \in G}|g| \eta(g)+\sum_{|g| \geq r^{1 / 2}}|g| \eta(g),
\end{aligned}
$$


which implies

$$
\frac{1}{r} \sum_{g:|g| \leq r}|g|^{2} \eta(g) \rightarrow 0 \text { as } r \rightarrow \infty .
$$

We conclude that $r \Lambda_{\eta}\left(e^{r}\right) \rightarrow 0$ as $r \rightarrow \infty$.

We now prove an explicit upper bound on entropy when the decay of the return probability $\mu^{(2 n)}(\mathrm{id})$ is much slower than $\exp \left(-n^{\frac{1}{2}}\right)$.

Theorem 3.2. Let $\mu$ be a symmetric probability measure of finite entropy on $G$. Suppose $\mu^{(2 n)}(\mathrm{id}) \geq \exp (-\gamma(n))$ where $\gamma:[1, \infty) \rightarrow \mathbb{R}_{+}$is a function such that for some $\beta \in\left(0, \frac{1}{2}\right)$, the function $n \rightarrow n^{\beta} / \gamma(n)$ is non-decreasing. Define

$$
\rho(n)=\inf \left\{x: \gamma^{-1}(x / 2) / x>n\right\} .
$$

Then there is a constant $C=C(\beta)$ such that

$$
H_{\mu}(n) \leq C(\rho(n)+1) .
$$

Proof. The proof is analogous to the proof of the "fundamental inequality", see for example [BHM08, Proposition 3.4].

Let

$$
k_{n}=\min \left\{k: \frac{e^{k}}{\gamma^{-1}\left(e^{k} / 2\right)}<\frac{1}{n}\right\}=\lceil\log \rho(n)\rceil .
$$

As before, let $\left(U_{k}\right)$ be a sequence of finite subsets in $G$ such that $\left|U_{k}\right|=e^{e^{k}}$ and $\lambda_{\mu}\left(U_{k}\right) \leq 2 \Lambda_{\mu}\left(e^{e^{k}}\right)$. Define $\Omega_{k}=\cup_{j=1}^{k} U_{j}^{-1} U_{j}, \Omega_{0}=\emptyset$. Note that from definition of the set $\Omega_{k}$, we have

$$
\left|\Omega_{k}\right| \leq \sum_{j=1}^{k}\left|U_{j}\right|^{2} \leq \frac{1}{1-e^{-2(e-1)}} e^{2 e^{k}}
$$

By (8) and (9), we have

$$
\mathbf{P}\left(W_{n} \notin \Omega_{k}\right) \leq \mathbf{P}\left(W_{n} \notin U_{k}^{-1} U_{k}\right) \leq \frac{n e^{k}}{4 \gamma^{-1}\left(e^{k} / 2\right)} .
$$

The entropy of $W_{n}$ can be decomposed as

$$
\begin{aligned}
H_{\mu}(n) & =\mathbf{P}\left(W_{n} \in \Omega_{k_{n}}\right) H\left(W_{n} \mid W_{n} \in \Omega_{k_{n}}\right) \\
& +\sum_{k=k_{n}+1}^{\infty} \mathbf{P}\left(W_{n} \in \Omega_{k} \backslash \Omega_{k-1}\right) H\left(W_{n} \mid W_{n} \in \Omega_{k} \backslash \Omega_{k-1}\right)+\Delta_{n},
\end{aligned}
$$


where

$\Delta_{n}=-\mathbf{P}\left(W_{n} \in \Omega_{k_{n}}\right) \log \left(\mathbf{P}\left(W_{n} \in \Omega_{k_{n}}\right)\right)-\sum_{k=k_{n}+1}^{\infty} \mathbf{P}\left(W_{n} \in \Omega_{k} \backslash \Omega_{k-1}\right) \log \left(\mathbf{P}\left(W_{n} \in \Omega_{k} \backslash \Omega_{k-1}\right)\right)$.

By convexity of entropy, we have

$$
\begin{gathered}
H_{\mu}(n)-\Delta_{n} \leq \log \left|\Omega_{k_{n}}\right|+\sum_{k \geq k_{n}+1} \log \left|\Omega_{k} \backslash \Omega_{k-1}\right| \mathbf{P}\left(W_{n} \in \Omega_{k} \backslash \Omega_{k-1}\right) \\
\leq c_{0}+2 e^{k_{n}}+\sum_{k \geq k_{n}} 2 e^{k+1} \mathbf{P}\left(W_{n} \notin \Omega_{k}\right),
\end{gathered}
$$

where $c_{0}=\log \frac{1}{1-e^{-2(e-1)}}$. By (14),

$$
\sum_{k \geq k_{n}} e^{k} \mathbf{P}\left(W_{n} \notin \Omega_{k}\right) \leq \sum_{k \geq k_{n}} e^{k} \frac{n e^{k}}{4 \gamma^{-1}\left(e^{k} / 2\right)} .
$$

From the assumption $n^{\beta} / \gamma(n)$ is non-decreasing and $\beta<\frac{1}{2}$, we have that $\gamma^{-1}(t) / \gamma^{-1}(s) \geq$ $(t / s)^{1 / \beta}$. Thus for all $k>k_{n}$,

$$
\frac{e^{2 k}}{\gamma^{-1}\left(e^{k} / 2\right)} \leq \frac{e^{2 k_{n}}}{\gamma^{-1}\left(e^{k_{n}} / 2\right)} \frac{e^{2 k-2 k_{n}}}{\left(e^{k} / e^{k_{n}}\right)^{1 / \beta}}=\frac{e^{2 k_{n}}}{\gamma^{-1}\left(e^{k_{n}} / 2\right)} e^{-(1 / \beta-2)\left(k-k_{n}\right)}
$$

Plug it into (15), we have

$$
\begin{aligned}
\sum_{k \geq k_{n}} e^{k} \mathbf{P}\left(W_{n} \notin \Omega_{k}\right) & \leq \frac{n e^{2 k_{n}}}{\gamma^{-1}\left(e^{k_{n}} / 2\right)} \sum_{k \geq k_{n}} e^{-(1 / \beta-2)\left(k-k_{n}\right)} \\
& \leq C_{\beta} \frac{n e^{2 k_{n}}}{\gamma^{-1}\left(e^{k_{n}} / 2\right)} \leq C_{\beta} e^{k_{n}}
\end{aligned}
$$

where $C_{\beta}=\left(1-e^{-(1 / \beta-2)}\right)^{-1}$. The last step used the definition of $k_{n}$. By the definition of $\rho(n)$, we have $\rho(n) \leq e^{k_{n}} \leq e \rho(n)$, we have that

$$
H_{\mu}(n)-\Delta_{n} \leq c_{0}+2\left(1+C_{\beta}\right) e^{k_{n}} \leq c_{0}+2 e\left(1+C_{\beta}\right) \rho(n) .
$$

Finally, we show that $\Delta_{n}$ is bounded by a constant. By the inequality $-a \log a \leq$ $2 e^{-1} \sqrt{a}$, we have

$$
\Delta_{n} \leq 2 e^{-1}+2 e^{-1} \sum_{k=k_{n}+1}^{\infty} \mathbf{P}\left(W_{n} \in \Omega_{k} \backslash \Omega_{k-1}\right)^{\frac{1}{2}}
$$


By (14) again, we have

$$
\begin{aligned}
\sum_{k=k_{n}+1}^{\infty} \mathbf{P}\left(W_{n}\right. & \left.\in \Omega_{k} \backslash \Omega_{k-1}\right)^{\frac{1}{2}} \leq \sum_{k=k_{n}+1}^{\infty}\left(\frac{n e^{k}}{4 \gamma^{-1}\left(e^{k} / 2\right)}\right)^{\frac{1}{2}} \\
& \leq\left(\frac{n e^{k_{n}}}{4 \gamma^{-1}\left(e^{\left.k_{n} / 2\right)}\right.}\right)^{\frac{1}{2}} \sum_{k=k_{n}+1}^{\infty} e^{-1 / 2(1 / \beta-1)\left(k-k_{n}\right)} \leq 2 C_{\beta} .
\end{aligned}
$$

We conclude that $\Delta_{n} \leq 2 e^{-1}\left(1+2 C_{\beta}\right)$. The statement follows from (16).

Remark 3.3. From the proof it is clear that Theorem 3.2 can be rephrased in terms of the spectral profile as follows. Let $\mu$ be a symmetric probability measure of finite entropy on $G$. Suppose there exists $\alpha>1$ such that $\frac{1}{x^{\alpha} \Lambda_{\mu}\left(e^{x}\right)}$ is non-decreasing. Define

$$
\tilde{\rho}(n)=\inf \left\{x: \Lambda_{\mu}\left(e^{x}\right) \leq \frac{1}{n}\right\}
$$

then there is a constant $C=C(\alpha)$ such that

$$
H_{\mu}(n) \leq C(\tilde{\varrho}(n)+1) .
$$

Remark 3.4. Suppose $\gamma(n)$ is a regularly varying function $\gamma(n)=n^{\beta} \ell(n)$ where $\beta \in(0,1 / 2)$, and $\ell$ is a slowly varying function satisfying $\ell\left(n^{b}\right) \simeq \ell(n)$ for all $b>0$. Then by standard asymptotic inversion, see [BGT87, Proposition 1.5.15], we have that the function $\rho$ defined in (13) satisfies

$$
\rho(n) \simeq n^{\frac{\beta}{1-\beta}} \ell^{\frac{1}{1-\beta}}(n) .
$$

In particular, Theorem 1.4 in the Introduction follows from Theorem 3.2 .

Example 3.5. Consider simple random walk on the wreath product $\mathbb{Z} \imath \mathbb{Z}$. By Pittet and Saloff-Coste $\mathrm{PSC02}$,

$$
\mu^{(2 n)}(e) \simeq \exp \left(-n^{\frac{1}{3}} \log ^{\frac{2}{3}} n\right) .
$$

From Erschler [Ers03], we have $H_{\mu}(n) \simeq n^{\frac{1}{2}} \log n$. Theorem 3.2 implies that $H_{\mu}(n) \leq$ $n^{\frac{1}{2}} \log n$, which is a sharp upper bound. We will see in Section 5 a family of groups where Theorem 3.2 provides sharp upper bounds on entropy.

\section{Upper bounds on rate of escape}

In this section, we show that if there is some additional information on the spectral profile of balls, then the Markov type inequality provides an upper bound on the rate of escape.

We first show that Lemma 3.1 can be strengthened to provide the following bound. 
Lemma 4.1. Let $\mu$ be a symmetric probability measure on $G$, and $U$ be a finite subset of $G$. Then for a $\mu$-random walk $\left(W_{n}\right)$ on $G$,

$$
\mathbf{P}\left(\exists k: 0 \leq k \leq n, W_{k} \notin U^{-1} U\right) \leq(32 n+1) \lambda_{\mu}(U) .
$$

Proof. Together with the classical Doob's $L^{2}$-maximal inequality for submartingles, the proof of the Markov type inequality (7) in [NP08, Theorem 2.1] yields the maximal inequality: for any $f \in Z^{1}(G, \pi)$ for some representation $\pi: G \rightarrow \operatorname{Isom}(\mathcal{H})$,

$$
\mathbf{E}\left[\max _{0 \leq k \leq n}\left\|f\left(W_{k}\right)\right\|_{\mathcal{H}}^{2}\right] \leq(32 n+1) \mathbf{E}\left[\left\|f\left(W_{1}\right)\right\|_{\mathcal{H}}^{2}\right] .
$$

To see this, let $v=\mathbf{E}\left[f\left(W_{1}\right)\right], X_{1}, X_{2}, \ldots$ be the random walk increments, then by [NP08, Equation (20)],

$$
2 f\left(W_{n}\right)=M_{n}-\pi\left(W_{n}\right) N_{n}+\pi\left(W_{n}\right) v,
$$

where $\left(M_{k}\right)_{k=0}^{n}$ is a martingle with respect to the filtration induced by $\left(X_{k}\right)_{k=0}^{n}$, and $\left(N_{k}\right)_{k=0}^{n}$ is a martingale with respect to $\left(X_{n-k}\right)_{k=0}^{n}$, and $M_{k}$ has the same law as $N_{k}$. Therefore

$$
\begin{aligned}
4 \mathbf{E}\left[\max _{0 \leq k \leq n}\left\|f\left(W_{k}\right)\right\|_{\mathcal{H}}^{2}\right] & \leq 3\left(\mathbf{E}\left[\max _{0 \leq k \leq n}\left\|M_{k}\right\|_{\mathcal{H}}^{2}\right]+\mathbf{E}\left[\max _{0 \leq k \leq n}\left\|N_{k}\right\|_{\mathcal{H}}^{2}\right]+\|v\|_{\mathcal{H}}^{2}\right) \\
& \leq 24 \mathbf{E}\left[\left\|M_{n}\right\|_{\mathcal{H}}^{2}\right]+3\|v\|_{\mathcal{H}}^{2} .
\end{aligned}
$$

In the last step we used Doob's $L^{2}$-maximal inequality for submartingales that $\mathbf{E}\left[\max _{0 \leq k \leq n}\left\|M_{k}\right\|_{\mathcal{H}}^{2}\right] \leq 4 \mathbf{E}\left[\left\|M_{n}\right\|_{\mathcal{H}}^{2}\right]$. By $[$ NP08, Equation (22)], we have

$$
\mathbf{E}\left[\left\|M_{n}\right\|_{\mathcal{H}}^{2}\right] \leq 4 n \mathbf{E}\left[\left\|f\left(W_{1}\right)\right\|_{\mathcal{H}}^{2}\right] .
$$

The inequality (17) follows.

Let the test function $\phi$ and the associated 1-coboundary $\sigma$ be chosen as in the proof of Lemma 3.1. Recall that if $g \notin U^{-1} U$, then supp $\phi \cap \operatorname{supp} \tau_{g} \phi=\emptyset$, and it follows that

$$
\|\sigma(g)\|_{2}^{2}=\|\phi\|_{2}^{2}+\left\|\tau_{g} \phi\right\|_{2}^{2}=2\|\phi\|_{2}^{2} .
$$

Therefore

$$
\begin{aligned}
2\|\phi\|_{2}^{2} \mathbf{P}\left(\exists k: 0 \leq k \leq n, W_{k} \notin U^{-1} U\right) & \leq \mathbf{E}\left[\max _{0 \leq k \leq n}\left\|\sigma\left(W_{k}\right)\right\|_{2}^{2}\right] \\
& \leq 2(32 n+1) \mathcal{E}_{\mu}(\phi) .
\end{aligned}
$$

The statement of the lemma follows from the choice of $\phi$. 
Let $\mu$ be a symmetric probability measure on $G$. Write $B(r)=B(\mathrm{id}, r)$. Consider the spectral profile of balls

$$
\lambda_{\mu}(B(r))=\inf \left\{\mathcal{E}_{\mu}(f): \operatorname{support}(f) \subset B(e, r),\|f\|_{2}=1\right\} .
$$

Theorem 4.2. Let $\mu$ be a symmetric probability measure on $G$. Suppose there exists a function $f$ and constant $\theta>0$ such that

$$
\lambda_{\mu}(B(r)) \leq f(r) \text { and } \frac{f(r)}{f(s)} \leq C_{0}\left(\frac{r}{s}\right)^{-\theta} \text { for all } r \geq s>0 .
$$

Then for any $\alpha \in(0, \theta)$, there exists a constant $C=C\left(\theta, \alpha, C_{0}\right)>0$ such that the $\mu$-random walk on $G$ satisfies

$$
\mathbf{E}\left[\max _{0 \leq k \leq n}\left|W_{k}\right|^{\alpha}\right] \leq C \varrho(n)^{\alpha}
$$

where

$$
\varrho(n):=\inf \left\{r>0: f(r)<\frac{1}{n}\right\}
$$

Proof. The proof is along the same lines as Theorem 3.2. Let $k_{n}=\inf \left\{k: f\left(e^{k}\right)<1 / n\right\}$, note that by definitions $e^{k_{n}-1}<\varrho(n) \leq e^{k_{n}}$. We have

$$
\mathbf{E} \max _{0 \leq k \leq n}\left|W_{k}\right|^{\alpha} \leq e^{\alpha\left(k_{n}+1\right)}+\sum_{k \geq k_{n}+1} e^{\alpha(k+1)} \mathbf{P}\left(\exists \ell \leq n: W_{\ell} \notin B\left(e^{k+1}\right) \backslash B\left(e^{k}\right)\right) .
$$

By Lemma 4.1, we have

$$
\mathbf{P}\left(\exists \ell \leq n: W_{\ell} \notin B\left(e^{k}\right)\right) \leq(32 n+1) \lambda_{\mu}\left(B\left(e^{k-1}\right)\right) .
$$

Therefore

$$
\begin{aligned}
\mathbf{E} \max _{0 \leq k \leq n}\left|W_{k}\right|^{\alpha} & \leq e^{\alpha k_{n}}+\sum_{k \geq k_{n}} e^{\alpha(k+1)}(32 n+1) \lambda_{\mu}\left(B\left(e^{k-1}\right)\right) \\
& \leq e^{\alpha\left(k_{n}+1\right)}+33 e^{2 \alpha} n \sum_{k \geq k_{n}} e^{\alpha k} f\left(e^{k}\right) \\
& =e^{\alpha\left(k_{n}+1\right)}+33 e^{2 \alpha} n f\left(e^{k_{n}}\right) \sum_{k \geq k_{n}} e^{\alpha k} \frac{f\left(e^{k}\right)}{f\left(e^{k_{n}}\right)} \\
& \leq e^{\alpha\left(k_{n}+1\right)}+e^{2 \alpha} C_{0} e^{\alpha k_{n}} \sum_{k \geq k_{n}} e^{-(\theta-\alpha)\left(k-k_{n}\right)} \\
& =\left(e^{\alpha}+\frac{C_{0} e^{2 \alpha}}{1-e^{-(\theta-\alpha)}}\right) e^{\alpha k_{n}} \\
& \leq C \rho(n)^{\alpha} .
\end{aligned}
$$


We now review some examples from the literature where symmetric simple random walks are known to satisfy $\lambda_{\mu}(B(r)) \simeq r^{-2}$. By the universal diffusive lower bound from Lee and Peres [LP13] and Theorem 1.6. we know that in these examples, symmetric simple random walks exhibit diffusive rate of escape, that is $L_{\mu}(n) \simeq n^{\frac{1}{2}}$.

Definition 4.3 (Tessera [Tes13]). The class $\mathcal{S}$ of finitely generated geometrically elementary solvable groups is the smallest class of finitely generated groups such that

(i) $\mathcal{S}$ contains all the finitely generated closed subgroups of $T(d, k)$ for any integer $d$ and local field $k$, where $T(d, k)$ is the group of invertible upper triangular matrices of size $d$ in a local field $k$;

(ii) $\mathcal{S}$ is stable under taking finite direct products, finitely generated subgroups and quotients;

(iii) $\mathcal{S}$ is stable under quasi-isometry.

The collection $\mathcal{S}$ is exactly the subset of finitely generated groups in the class GES in Tes13. Our results actually extend to compactly generated locally compact unimodular groups, but for simplicity we restrict ourselves to discrete groups here.

Example 4.4. The following examples are known to be in $\mathcal{S}$, see Tessera [Tes13].

- finitely generated nilpotent groups, more generally polycyclic groups,

- lamplighter groups $F(\mathbb{Z}$, where $F$ is a finite group,

- solvable Baumslag-Solitar groups $B S(1, q)=\left\langle t, x \mid t x t^{-1}=x^{q}\right\rangle$.

- torsion free solvable groups of finite Prüfer rank. Recall that a group has finite Prüfer rank if there is an integer $r$ such that any of its finitely generated subgroup admits a generating set of cardinality less or equal to $r$. Return probability and the spectral profile of simple random walks on torsion free solvable groups of finite Prüfer rank are also treated in Pittet and Saloff-Coste [PSC03], where the authors proved the lower bound $\mu^{(2 n)}(\mathrm{id}) \gtrsim e^{-n^{1 / 3}}$ for $\mu$ a symmetric probability measure with finite generating support on the group.

Note that the class $\mathcal{S}$ is defined in both algebraic and geometric terms. It contains non-virtually solvable groups such as $F \imath \mathbb{Z}$ where $F$ is a non-solvable finite group. It also contains finitely generated groups that are not residually finite, in particular not linear, see [Tes13].

Corollary 4.5. Let $G$ be a group in the class $\mathcal{S}$. Then for any symmetric probability measure $\mu$ with finite second moment on $G$, there exists a constant $C=C(\mu, G)$ such that for any $\alpha \in[1,2)$,

$$
\mathbf{E} \max _{0 \leq k \leq n}\left|W_{k}\right|^{\alpha} \leq \frac{C}{2-\alpha} n^{\frac{\alpha}{2}} .
$$




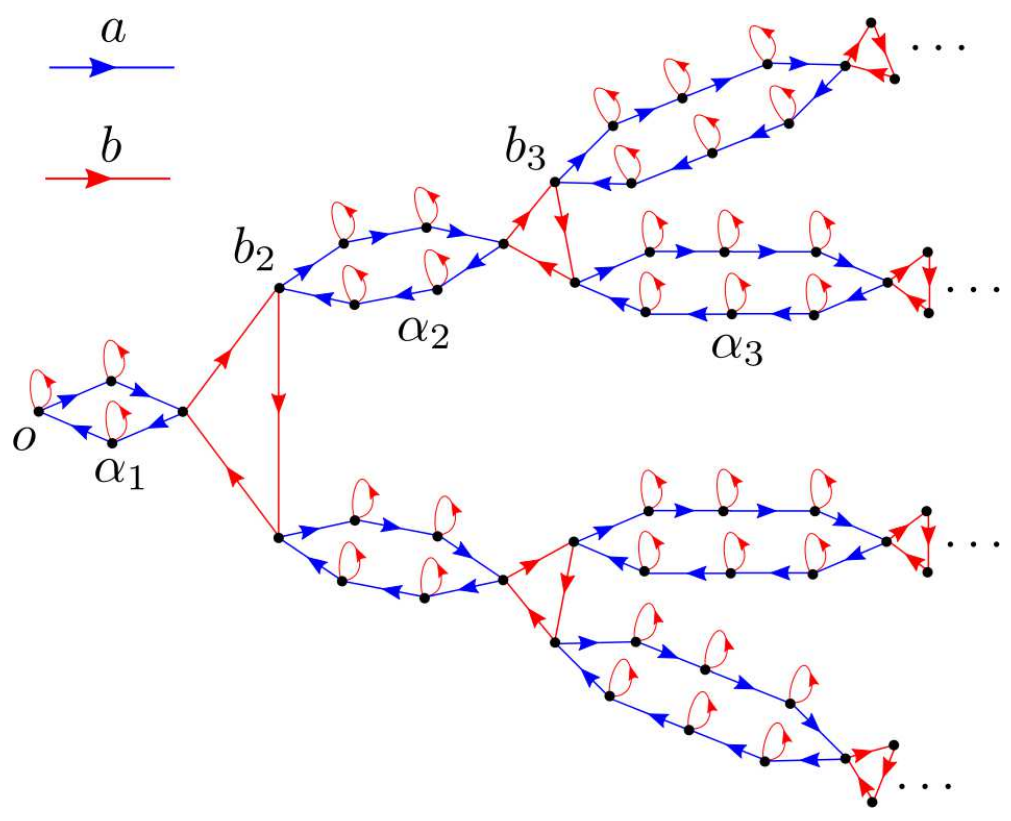

Figure 1: the first 3 levels of the Schreier graph, picture from M. Kotowski and B. Virág [KV15]

Proof. The main result of [Tes13] states that on a geometrically elementary solvable group, $\lambda_{\mu}(B(r)) \simeq r^{-2}$ for a symmetric probability measure $\mu$ of finite generating support. By standard comparison of Dirichlet forms [PSC00], the same estimates holds for a symmetric probability measures $\mu^{\prime}$ with finite second moment. Then Theorem 4.2 applies.

\section{Sharpness of the entropy bounds}

To illustrate the sharpness of the bound in Theorem 3.2 when the decay of return probability is away from the critical behavior $\exp \left(-n^{\frac{1}{2}}\right)$, we consider the family of bubble groups as in Kotowski and Virág [KV15]. The bubble groups were first introduced by Amir and Kozma [AK16] in their study of growth of harmonic functions on groups.

The bubble groups are defined via their actions on certain marked graphs. We first describe these graphs. Let $\mathbf{a}=\left(\alpha_{1}, \alpha_{2}, \ldots\right)$ be a sequence of natural numbers, $\mathbf{T}$ be a rooted tree with forward degree sequence $(1,2,2, \ldots)$, that is except the root, every vertex has two children. The bubble graph $\mathcal{M}_{\mathbf{a}}$ is obtained as follows. Each edge at level $k \geq 1$ (we make the convention that the level of an edge is the level of the child on that edge) is replaced by a cycle of length $2 \alpha_{k}$ called a bubble. Each vertex at level $k \geq 1$ (we ignore the root which is now part of a cycle of length 
$2 \alpha_{1}$ ) is blown-up to a 3 -cycle. These 3 -cycles are called branching cycles. Finally, at each vertex which belongs only to a bubble (but not to a branching cycle), we add a self loop. Having chosen an orientation along each cycle (say, clockwise), we label each edge of the bubble with the letter $a$, each edge of the branching cycle with the letter $b$, and the self loops at vertices that belong only to a bubble are labeled with the letter $b$. Figure 1 represents the first 3 levels of the Schreier graph $\mathcal{M}_{\mathbf{a}}$, with $\alpha_{1}=2, \alpha_{2}=3$ and $\alpha_{3}=4$.

The bubble group $\Gamma_{\mathbf{a}}$ is a subgroup of the permutation group of the vertex set of $X_{\mathbf{a}}$ generated by two elements $a$ and $b$. The action of the permutation $a$ (resp. $b$ ) on any vertex $x$ in $\mathcal{M}_{\mathbf{a}}$ is indicated by the oriented labeled edge at $x$ marked with an $a$ (resp. a $b$ ). Informally, $a$ rotates the long bubbles whereas $b$ rotates the branching cycles. We write the left group action of $g$ on vertex $x$ as $g \cdot x$.

Consider the permutational wreath product of the bubble group $\Gamma_{\mathbf{a}}$ with $\mathbb{Z}$ over the Schreier graph $\mathcal{M}_{\mathbf{a}}, G_{\mathbf{a}}=\mathbb{Z} \imath_{\mathcal{M}_{\mathbf{a}}} \Gamma_{\mathbf{a}}$. Formally, $G$ is the semi-direct product $\left(\oplus_{\mathcal{M}_{\mathbf{a}}} \mathbb{Z}\right) \rtimes \Gamma_{\mathbf{a}}$, where $\Gamma_{\mathbf{a}}$ acts by permuting the coordinates. A group element of $G$ is recorded as $(f, \gamma)$, where $f: \mathcal{M}_{\mathbf{a}} \rightarrow \mathbb{Z}$ is a function of finite support, and $\gamma \in \Gamma_{\mathbf{a}}$. Multiplication is given by

$$
(f, \gamma)\left(f^{\prime}, \gamma^{\prime}\right)=\left(f+\tau_{\gamma} f^{\prime}, \gamma \gamma^{\prime}\right)
$$

where $\tau_{\gamma}$ is the translation $\tau_{\gamma} f(x)=f\left(\gamma^{-1} \cdot x\right)$ and we use additive notation for $\mathbb{Z}$. We identify $\Gamma_{\mathbf{a}}$ as a subgroup of $G_{\mathbf{a}}$ by the embedding $\gamma \mapsto(\mathbf{0}, \gamma)$ for every $\gamma \in \Gamma_{\mathbf{a}}$ and $\mathbb{Z}$ as a subgroup of $G_{\mathbf{a}}$ by $z \mapsto\left(z \delta_{0}, \operatorname{id}_{\Gamma_{\mathbf{a}}}\right)$.

Let $\nu$ be the symmetric probability measure on $\Gamma_{\mathbf{a}}$ defined as

$$
\nu\left(a^{ \pm 1}\right)=\nu\left(b^{ \pm 1}\right)=\frac{1}{4} .
$$

Let $\eta$ be the symmetric probability measure on $\mathbb{Z}$ defined as $\eta( \pm 1)=\frac{1}{2}$. Consider the "switch-walk-switch" measure $\mu=\eta * \nu * \eta$ on the permutational wreath product $G_{\mathbf{a}}$. The $\mu$-random walk on $G_{\mathbf{a}}$ can be described as follows. Let $X_{1}, X_{2}, \ldots$ be a sequence of i.i.d. random variables on $\Gamma_{\mathbf{a}}$ with distribution $\nu$ and $Z_{1}, Z_{2}, \ldots$ be a sequence of i.i.d. random variables on $\mathbb{Z}$ with distribution $\eta$. Then the random variable $W_{n}=Z_{1} X_{1} Z_{2} \ldots Z_{2 n-1} X_{n} Z_{2 n}$ has distribution $\mu^{(n)}$. Write $W_{n}=\left(L_{n}, Y_{n}\right)$, where $L_{n}$ denotes the lamp configuration at time $n$ and $Y_{n}$ denotes the position of the walker on the Cayley graph of $\Gamma_{\mathbf{a}}$. Then we have

$$
\begin{aligned}
Y_{n} & =X_{1} \ldots X_{n}, \\
L_{n}(x) & =Z_{1} \mathbf{1}_{\{x=o\}}+\sum_{j=1}^{n-1}\left(Z_{2 j}+Z_{2 j+1}\right) \mathbf{1}_{\left\{x=Y_{j} \cdot o\right\}}+Z_{2 n} \mathbf{1}_{\left\{x=Y_{n} \cdot o\right\}} .
\end{aligned}
$$

An important point is that the $\mathbb{Z}$-lamp configurations are updated along the inverted orbit $\left(Y_{j} \cdot o\right)_{j=0}^{n}$. More information about permutational wreath products and random walks with "switch-walk-switch" step distribution on them can be found in [AV12, KV15] . 
Whether $H_{\mu}(n)$ grows linearly or not is closely related to the transience/recurrence property of the underlying bubble graph. Indeed, when the induced random walk $\left(Y_{n}^{-1} \cdot o\right)$ is transient on $\mathcal{M}_{\mathbf{a}}$, the Poisson boundary of $(G, \mu)$ is non-trivial, see [KV15, Theorem 5.1] and also Bartholdi and Erschler [BE16, Section 3]. Note that on the bubble graph, the effective resistance from the root $o$ to the level $k$ branching cycles is quite easy to calculate. The graph $\mathcal{M}_{\mathbf{a}}$ is recurrent if and only if

$$
\sum_{k=1}^{\infty} \frac{\alpha_{k}}{2^{k}}=\infty
$$

We now apply the method in Amir and Virág AV12 to give an explicit entropy lower bound for the $\mu$-random walk on $\mathbb{Z} \imath_{\mathcal{M}_{\mathbf{a}}} \Gamma_{\mathbf{a}}$, in the case that $\inf _{k} \alpha_{k+1} / \alpha_{k}>2$. The basic idea for the lower bound is to collect contribution to entropy from the lamp configurations over the Schreier graph. The permutational wreath extension with $\mathbb{Z}$ plays an important role in both the entropy lower bound and return probability upper bound. Let $Q_{n}$ be the occupation time measure of the inverted orbit $\left(Y_{j} \cdot o\right)_{j=0}^{n}$ in the first $n$ steps. Namely, for each $x \in \mathcal{M}_{\mathbf{a}}, Q_{n}(x)$ denotes the number of visits of the inverted orbit to the vertex $x$ in the first $n$ steps,

$$
Q_{n}(x)=\sum_{j=0}^{n} \mathbf{1}_{\left\{x=Y_{j} \cdot \circ\right\}} .
$$

Lemma 5.1. Let a be a scaling sequence satisfying

$$
\inf _{k} \alpha_{k+1} / \alpha_{k}>2
$$

Then for the $\mu$-random walk on $G_{\mathbf{a}}$ described above, there exists a constant $c>0$ such that

$$
H_{\mu}(n) \geq c\left|B_{\mathcal{M}_{\mathbf{a}}}(o, \sqrt{n})\right| \log n .
$$

Proof. Denote by $T$ the first time that the induced random walk returns to $o$,

$$
T=\min \left\{n>0: Y_{n} \cdot o=o\right\}=\min \left\{n>0: Y_{n}^{-1} \cdot o=o\right\} .
$$

For simple random walk $\nu$ on $\mathbb{Z}$ we have $H_{\nu}(k) \geq c_{0} \log k$. Let $Q_{n}$ be defined as in (18), then

$$
H_{\mu}(n) \geq H\left(W_{n} \mid Q_{n}\right) \geq c_{0} \mathbf{E}\left[\sum_{x \in X_{\mathbf{a}}} \log \left(1+Q_{n}(x)\right)\right] .
$$

From the proof of [AV12, Theorem 9], we have that for any sub-additive function $f:[0, \infty) \rightarrow[0, \infty)$,

$$
\mathbf{E}\left[\sum_{x \in X_{\mathbf{a}}} f\left(Q_{n}(x)\right)\right] \geq \frac{n p f\left(\frac{1}{p}\right)}{16}
$$


for all $0<p \leq \mathbf{P}(T>n)$. Next we apply (20) with $f(x)=\log (1+x)$. By (19), a lower estimate on $\mathbf{P}(T>n)$ will give a lower bound for entropy. We now follow the idea in the proof of [AV12, Proposition 18].

Let $S_{r}=\left\{x \in \mathcal{M}_{\mathbf{a}}: d(o, x)=r\right\}$ be the set of vertices that are distance $r$ from the root $o$. Let $\sigma_{r}$ denote the hitting time of the set $S_{r}$, and $T_{r}^{*}$ be the first return to $o$ after hitting $S_{r}$, that is $T_{r}^{*}=\inf \left\{n: Y_{n}^{-1} \cdot o=o, n>\sigma_{r}\right\}$. Then on the event that the inverted orbit $\left\{Y_{j}^{-1} \cdot o\right\}_{j \geq 1}$ hits $S_{r}$ before $o$ and the first return time to $o$ after hitting $S_{r}$ is later than $n$, we have the first return to $o$ is after time $n$. In other words we have inclusion of events:

$$
\{T>n\} \supseteq\left\{\left\{Y_{j}^{-1} \cdot o\right\}_{j \geq 1} \text { hits } S_{r} \text { before } o\right\} \cap\left\{T_{r}^{*}>n\right\} .
$$

Let $\mathcal{R}\left(o \leftrightarrow S_{r}\right)$ be the effective resistance between the root $o$ and the level set $S_{r}$, then

$$
\mathbf{P}\left(\left\{Y_{j}^{-1} \cdot o\right\}_{j \geq 1} \text { hits } S_{r} \text { before } o\right)=\frac{1}{2 \mathcal{R}\left(o \leftrightarrow S_{r}\right)} .
$$

We have for $r=\ell+\sum_{j=1}^{k} \alpha_{j}+k$, where $0 \leq \ell \leq \alpha_{k+1}$,

$$
\mathbf{P}\left(\left\{Y_{j}^{-1} \cdot o\right\}_{j \geq 1} \text { hits } S_{r} \text { before } o\right)=\frac{1}{2\left(2^{-k-1} \ell+\sum_{j=1}^{k} 2^{-j} \alpha_{j}\right)} \geq \frac{1}{2^{-k+2}\left(\alpha_{k}+\ell\right)} .
$$

In the last step we used the assumption that $\inf _{k} \alpha_{k+1} / \alpha_{k}>2$.

Let $\tilde{\sigma}_{\ell}$ denote the hitting time of 0 of lazy simple random walk on the interval $[0, \ell] \cap \mathbb{Z}$ with reflecting boundary, starting from the end $\ell$. Then $T_{r}^{*}$ stochastically dominates $\tilde{\sigma}_{\ell}+\tilde{\sigma}_{\alpha_{k}}+\ldots+\tilde{\sigma}_{\alpha_{1}}$. Therefore from classical estimates of lazy simple random walk on an interval, we have that there exists an absolute constant $c_{1}>0$ such that

$$
P\left(T_{r}^{*}>c_{1}\left(\alpha_{k}^{2}+\ell^{2}\right)\right) \geq \frac{1}{4}
$$

From the Markov property, we have

$$
P\left(T>c\left(\alpha_{k}^{2}+\ell^{2}\right)\right) \geq \frac{1}{4} \frac{1}{2^{-k+2}\left(\alpha_{k}+\ell\right)}=\frac{2^{k}}{16\left(\alpha_{k}+\ell\right)} .
$$

For time $n=c\left(\alpha_{k}^{2}+\ell^{2}\right)$, take $p_{n}=\frac{2^{k}}{16\left(\alpha_{k}+\ell\right)}$, by (20)

$$
H_{\mu}(n) \geq c_{0} \mathbf{E}\left[\sum_{x \in X_{\mathbf{a}}} \log \left(1+Q_{n}(x)\right)\right] \geq \frac{c_{0} n p_{n} \log \left(1+\frac{1}{p_{n}}\right)}{16} .
$$

Therefore for $n \in\left(c \alpha_{k}^{2}, c \alpha_{k}^{2}+c \alpha_{k+1}^{2}\right)$,

$$
H_{\mu}(n) \geq c_{0}^{\prime} n\left(\frac{2^{k}}{\sqrt{n}}\right) \log \left(\frac{\sqrt{n}}{2^{k}}\right) .
$$


Note that the volume of the ball $B_{\mathcal{M}_{\mathbf{a}}}(o, \sqrt{n})$ is comparable to $2^{k} \sqrt{n}$ for $n \in$ $\left(c \alpha_{k}^{2}, c \alpha_{k}^{2}+c \alpha_{k+1}^{2}\right)$, the statement follows from (22).

The spectral profile $\Lambda_{\mu, G_{\mathbf{a}}}$ is estimated in [SCZ15, Theorem 5.8]. In particular, if there exists a constant $\theta>1$ such that the scaling sequence a satisfies

$$
\inf _{k} \frac{\alpha_{k+1}}{\alpha_{k}} \geq \theta
$$

then there exists a constant $C=C(\theta)>0$ such that

$$
\frac{1}{C r^{2}} \leq \Lambda_{\mu, G_{\mathbf{a}}}\left(\left|B_{\mathcal{M}_{\mathbf{a}}}(o, r)\right| !\right) \leq \frac{C}{r^{2}}
$$

It follows from the Coulhon-Grigor'yan theory [Cou96, CG97] that in this case, the decay of the return probability satisfies

$$
\mu^{(2 n)}(\mathrm{id}) \simeq \exp \left(-\frac{n}{\phi(n)^{2}}\right)
$$

where $\phi$ is the inverse of the function $r \rightarrow r^{2}\left|B_{\mathcal{M}_{\mathbf{a}}}(o, r)\right| \log r$.

In the special case where $\theta>2$, we have

$$
\frac{n}{\phi(n)^{2}} \leq n^{\frac{\theta+1}{3 \theta+1}}(\log n)^{\frac{2 \theta}{3 \theta+1}} .
$$

Therefore the assumptions of Theorem 3.2 are satisfied, we can deduce an entropy upper bound from (23). In fact it is easier to use the upper bound on the spectral profile directly, see Remark 3.3. From the bound $\Lambda_{\mu, G_{\mathbf{a}}}\left(\left|B_{\mathcal{M}_{\mathbf{a}}}(o, r)\right| !\right) \leq \frac{C}{r^{2}}$, we have that

$$
\tilde{\rho}(n)=\inf \left\{x: \Lambda_{\mu}\left(e^{x}\right) \leq \frac{1}{n}\right\} \leq \log \left(\left|B_{\mathcal{M}_{\mathbf{a}}}(o, \sqrt{C n})\right| !\right) .
$$

Therefore by Theorem 3.2, we have

$$
H_{\mu}(n) \leq \log \left(\left|B_{\mathcal{M}_{\mathbf{a}}}(o, \sqrt{C n})\right| !\right) \leq C^{\prime}\left|B_{\mathcal{M}_{\mathbf{a}}}(o, \sqrt{n})\right| \log n .
$$

Comparing the bound to Lemma 5.1, we see that when the scaling sequence a satisfies $\inf _{k} \alpha_{k+1} / \alpha_{k}>2$, the entropy upper bound deduced from return probability by Theorem 3.2 is sharp.

Proof of Proposition 1.8 . The wreath product $\mathbb{Z} \imath \mathbb{Z}$ satisfies the statement with $\beta=$ $1 / 3$.

Take $\alpha_{k}=2^{\theta k}$ with $\theta \in(1, \infty)$ as the scaling sequence in the bubble graph $\mathcal{M}_{\mathbf{a}}$. Then by [SCZ15, Theorem 5.8], see also [SCZ15, Example 5.11], on the group $G_{\mathbf{a}}=\mathbb{Z} \imath_{\mathcal{M}_{\mathbf{a}}} \Gamma_{\mathbf{a}}$, we have

$$
\mu^{(2 n)}(\mathrm{id}) \simeq \exp \left(-n^{\frac{\theta+1}{3 \theta+1}}(\log n)^{\frac{2 \theta}{3 \theta+1}}\right) .
$$


By Lemma 5.1, we have

$$
H_{\mu}(n) \geq \frac{c(\theta-1)}{2 \theta} n^{\frac{\theta+1}{2 \theta}} \log n .
$$

Using Theorem [3.2, we obtain an upper bound on $H_{\mu}(n)$ from the lower bound on $\mu^{(2 n)}(\mathrm{id})$, it matches the entropy lower bound above.

\section{Extension to random walks on transitive graphs}

In this section we explain how to extend Theorem 1.1, 1.4 and 1.6 to symmetric random walks on transitive graphs. Let $X=(V, E)$ be a vertex transitive graph, which is locally finite, infinite and connected. By $\operatorname{Aut}(X)$ we denote the full automorphism group of $X$. The group $\operatorname{Aut}(X)$ is locally compact, equipped with the topology of pointwise convergence. In what follows, let $\Gamma$ be a closed subgroup of $\operatorname{Aut}(X)$ that acts transitively on $X$.

We say a Markov operator $P$ on $X$ is space homogenous if it is invariant under $\Gamma$, that is,

$$
P(g \cdot x, g \cdot y)=P(x, y) \text { for all } g \in \Gamma, x, y \in X .
$$

Further we may assume that $P$ is irreducible. The Poisson boundary and entropy theory of space homogenous Markov chains was systematically developed in Kaimanovich and Woess [KW02]. Let $H_{P}(n)$ be the Shannon entropy of the $n$-step transition probability,

$$
H_{P}(n)=-\sum_{x \in V} P^{n}(o, x) \log P^{n}(o, x) .
$$

By [KW02, for transition operator of finite entropy $H_{P}(1)<\infty$, the asymptotic entropy $h(P)=\lim _{n \rightarrow \infty} H_{P}(n) / n$ exists, and $(X, P)$ has the Liouville property if and only if $h(P)=0$.

Fix a reference point $o \in X$. We will only consider symmetric Markov operators, $P(x, y)=P(y, x)$ for all $x, y \in V$.

Theorem 6.1. Let $P$ be a space homogenous symmetric Markov chain on $X$ such that $H_{P}(1)<\infty$. Theorem 1.1 and 3.2 hold with $\mu^{(2 n)}(\mathrm{id})$ replaced by $P^{(2 n)}(o, o)$ and $H_{\mu}(n)$ replaced by $H_{P}(n)$.

For each vertex $x \in X$, let $\Gamma_{x}$ be its stabilizer, $\Gamma_{x}=\{g \in \Gamma, g \cdot x=x\}$. Write $K=\Gamma_{o}$. Since $K$ is open and compact, we can normalize the left-invariant Haar measure $m_{\Gamma}$ on $\Gamma$ such that $m_{\Gamma}(K)=1$. Denote by $\hat{m}_{\Gamma}$ the involution of $m_{\Gamma}$, that is $\hat{m}_{\Gamma}(A)=m_{\Gamma}\left(A^{-1}\right)$. The modular function $\Delta$ of the group $\Gamma$ is defined as

$$
\Delta(g)=\Delta_{\Gamma}(g)=\frac{d m_{\Gamma}}{d \hat{m}_{\Gamma}}(g)
$$


We say the group $\Gamma$ is unimodular if $\Delta \equiv 1$.

For a vertex $x$ in the graph $X$, consider the coset of $K$ in $\Gamma$ that moves $o$ to $x,\{g \in \Gamma, g \cdot o=x\}=g_{x} K$, where $g_{x} \cdot o=x$. Since the graph $X$ is assumed to be locally finite and connected, we have that $\left\{g_{x} K: x \sim o\right.$ or $\left.x=o\right\}$ generates the group $\Gamma$.

By [KW02, Proposition 2.15], there is a one-to-one correspondence between $\Gamma$ invariant Markov operators on $X$ and bi- $K$-invariant probability measures on $\Gamma$. Namely, given a bi- $K$-invariant probability measure $\mu$ on $\Gamma$, define $P_{\mu}$ as

$$
P_{\mu}(g \cdot o, h \cdot o)=\mu\left(g^{-1} h K\right)
$$

then $P_{\mu}$ is a $\Gamma$-invariant Markov operator on $X$. Note that $P_{\mu}$ is well-defined because $\mu$ is bi- $K$-invariant. In the other direction, set

$$
d \mu(g)=P(o, g \cdot o) d m_{\Gamma}(g),
$$

then $P=P_{\mu}$. This correspondence allows one to lift a $\Gamma$-invariant Markov chain on $X$ to a random walk on $\Gamma$ with step distribution $\mu$. Recall that $d m_{\Gamma}$ is normalized such that $m_{\Gamma}(K)=1$. Let $d \nu_{K}(g)=d m_{\Gamma}(g) \mathbf{1}_{\{g \in K\}}$ be the restriction of $m_{\Gamma}$ to $K$. Then $\nu_{K}$ is the normalized Haar measure on the compact group $K$. Note that since $\mu$ is bi-K-invariant, $\nu_{K} * \mu^{(n)}=\mu^{(n)}$ and it is bi-K-invariant. Then by the formula (24), we have that

$$
P^{n}(o, x)=\frac{d \mu^{(n)}}{d m_{\Gamma}}\left(g_{x}\right), \text { for any } g_{x} \text { such that } g_{x} \cdot o=x .
$$

It follows that for entropy we have the identity

$$
H_{P}(n)=-\int_{\Gamma} \log \left(\frac{d \mu^{(n)}}{d m_{\Gamma}}(g)\right) d \mu^{(n)}(g) .
$$

We now focus on $\Gamma$-invariant Markov chains on $X$ that are symmetric, $P(x, y)=$ $P(y, x)$ for all $x, y \in V$. Consider the associated Dirichlet form

$$
\mathcal{E}_{P}(f)=\frac{1}{2} \sum_{x, y \in G}(f(x)-f(y))^{2} P(x, y)
$$

and define

$$
\lambda_{P}(\Omega)=\inf \left\{\mathcal{E}_{P}(f): \operatorname{support}(f) \subset \Omega,\|f\|_{\ell^{2}(X)}=1\right\} .
$$

One can define the spectral profile $\Lambda_{P}$ in the same way as before. Let $\rho(P)=$ $\sup _{f \neq \mathbf{0}} \frac{\|P f\|_{\ell^{2}(X)}}{\|f\|_{\ell^{2}(X)}}$ be the spectral radius of $P$. A fundamental result due to Soardi and Woess [SW90] states that the spectral radius of an irreducible, $\Gamma$-invariant and symmetric Markov operator $P$ is 1 if and only if $\Gamma$ is both amenable and unimodular. 
Proof of Theorem [6.1. The assumption on the sub-exponential decay of $P^{n}(o, o)$ implies that $\rho(P)=1$, therefore by [SW90] we have that $\Gamma$ is both amenable and unimodular.

Note that when $\Gamma$ is unimodular, a symmetric $\Gamma$-invariant Markov operator $P$ lifts to a symmetric probability measure on $\Gamma$. Indeed, by (25),

$$
\begin{aligned}
d \mu\left(g^{-1}\right) & =P\left(o, g^{-1} \cdot o\right) d m_{\Gamma}\left(g^{-1}\right)=P\left(g^{-1} \cdot o, o\right) d m_{\Gamma}\left(g^{-1}\right) \quad \text { (symmetry) } \\
& \left.=P(o, g \cdot o) d m_{\Gamma}\left(g^{-1}\right) \quad \text { (invariance under } \Gamma\right) \\
& =P(o, g \cdot o) d m_{\Gamma}(g) \quad \text { (unimodularity) } \\
& =d \mu(g) .
\end{aligned}
$$

The Coulhon-Grigor'yan theory that relates the spectral profile of $P$ to the decay of $P^{(2 n)}(o, o)$ works for more general reversible random walks on graphs, see [Cou96]. In particular, Lemma 2.1 is valid with $\mu^{(2 n)}$ (id) replaced by $P^{2 n}(o, o)$ and $\Lambda_{\mu}$ replaced by $\Lambda_{P}$. Note also that the Markov type inequality (77) holds for general symmetric probability measure $\mu$ on a locally compact group $G$.

Given a finite set $U$ in the graph $X$, we can lift $U$ up to a set $\tilde{U}$ in $\Gamma$, that is

$$
\tilde{U}=\cup\left\{g_{x} K, g_{x} \cdot o \in U\right\} .
$$

Denote by $\tilde{W}_{n}$ a random walk on $\Gamma$ with step distribution $\mu$. Lemma 3.1 holds for $\tilde{W}_{n}$ :

$$
\mathbf{P}\left(W_{n} \notin \tilde{U}^{-1} \tilde{U}\right) \leq n \lambda_{\mu}(\tilde{U}) \leq n \lambda_{P}(U) .
$$

It is crucial here that $\mu$ is a symmetric probability measure on $\Gamma$, which is a consequence of unimodularity of $\Gamma$. Then as in Section 3, the proof of Theorem 1.1 and 3.2 goes through for $(G, \mu)$ and yields upper estimates for $H_{\mu}(n)$ as stated. By the identity (26), the same conclusions hold for $H_{P}(n)$.

Finally, we remark that Theorem 4.2 holds for a space homogenous symmetric Markov operator $P$ with $\lambda_{\mu}(B(\mathrm{id}, r))$ replaced by $\lambda_{P}(B(o, r))$, and the distance $|\cdot|$ taken to be the graph distance on $X$. The proof follows the same procedure of lifting $P$ to a probability measure on $\Gamma$. Again, the assumption on the decay of the spectral profile of balls implies that $\rho(P)=1$, therefore the measure $\mu$ on $\Gamma$ is symmetric.

Acknowledgements. We thank Michał Kotowski and Balint Virág for providing Figure 1, and Ryokichi Tanaka for reading the manuscript carefully and giving helpful comments. We thank the anonymous referees for comments that improved the paper.

\section{References}

[AK16] Gideon Amir and Gady Kozma. Groups with minimal harmonic functions as small as you like. arXiv preprint arXiv:1605.07593, 2016. 
[Ale92] G. Alexopoulos. A lower estimate for central probabilities on polycyclic groups. Canad. J. Math., 44(5):897-910, 1992.

[ANP09] Tim Austin, Assaf Naor, and Yuval Peres. The wreath product of $\mathbb{Z}$ with $\mathbb{Z}$ has Hilbert compression exponent $\frac{2}{3}$. Proc. Amer. Math. Soc., 137(1):85-90, 2009.

[AV12] Gideon Amir and Bálint Virág. Speed exponents of random walks on groups. To appear in International Mathematics Research Notices, arXiv:1203.6226, 2012.

[Ave76] A. Avez. Harmonic functions on groups. In Differential geometry and relativity, pages 27-32. Mathematical Phys. and Appl. Math., Vol. 3. Reidel, Dordrecht, 1976.

[BE16] Laurent Bartholdi and Anna Erschler. Poisson-furstenberg boundary and growth of groups. Probability Theory and Related Fields, pages 1-26, 2016.

[BGT87] N. H. Bingham, C. M. Goldie, and J. L. Teugels. Regular variation, volume 27 of Encyclopedia of Mathematics and its Applications. Cambridge University Press, Cambridge, 1987.

[BHM08] Sébastien Blachère, Peter Haïssinsky, and Pierre Mathieu. Asymptotic entropy and Green speed for random walks on countable groups. Ann. Probab., 36(3):1134-1152, 2008.

[CG97] Thierry Coulhon and Alexander Grigor'yan. On-diagonal lower bounds for heat kernels and Markov chains. Duke Math. J., 89(1):133-199, 1997.

[CGP01] T. Coulhon, A. Grigor'yan, and C. Pittet. A geometric approach to ondiagonal heat kernel lower bounds on groups. Ann. Inst. Fourier (Grenoble), 51(6):1763-1827, 2001.

[Cou96] Thierry Coulhon. Ultracontractivity and Nash type inequalities. J. Funct. Anal., 141(2):510-539, 1996.

[dCTV07] Yves de Cornulier, Romain Tessera, and Alain Valette. Isometric group actions on Hilbert spaces: growth of cocycles. Geom. Funct. Anal., 17(3):770-792, 2007.

[Der80] Yves Derriennic. Quelques applications du théorème ergodique sousadditif. 74:183-201, 4, 1980.

[Ers03] Anna Erschler. On drift and entropy growth for random walks on groups. Ann. Probab., 31(3):1193-1204, 2003.

[Ers06] Anna Erschler. Piecewise automatic groups. Duke Math. J., 134(3):591613, 2006. 
[GK04] Erik Guentner and Jerome Kaminker. Exactness and uniform embeddability of discrete groups. J. London Math. Soc. (2), 70(3):703-718, 2004.

[Gou16] Antoine Gournay. The Liouville property and Hilbertian compression. Ann. Inst. Fourier (Grenoble), 66(6):2435-2454, 2016.

[Kai91] Vadim A. Kaimanovich. Poisson boundaries of random walks on discrete solvable groups. In Probability measures on groups, X (Oberwolfach, 1990), pages 205-238. Plenum, New York, 1991.

[KV83] V. A. Kaŭmanovich and A. M. Vershik. Random walks on discrete groups: boundary and entropy. Ann. Probab., 11(3):457-490, 1983.

[KV15] Michał Kotowski and Bálint Virág. Non-Liouville groups with return probability exponent at most 1/2. Electron. Commun. Probab., 20:no. 12,2015 .

[KW02] Vadim A. Kaimanovich and Wolfgang Woess. Boundary and entropy of space homogeneous Markov chains. Ann. Probab., 30(1):323-363, 2002.

[LP13] James R Lee and Yuva Peres. Harmonic maps on amenable groups and a diffusive lower bound for random walks. The Annals of Probability, 41(5):3392-3419, 2013.

[LP16] Russell Lyons and Yuval Peres. Probability on Trees and Networks. Cambridge University Press, 2016.

[NP08] Assaf Naor and Yuval Peres. Embeddings of discrete groups and the speed of random walks. Int. Math. Res. Not. IMRN, 2008.

[PSC00] Ch. Pittet and L. Saloff-Coste. On the stability of the behavior of random walks on groups. J. Geom. Anal., 10(4):713-737, 2000.

[PSC02] C. Pittet and L. Saloff-Coste. On random walks on wreath products. Ann. Probab., 30, no.2:948-977, 2002.

[PSC03] Ch. Pittet and L. Saloff-Coste. Random walks on finite rank solvable groups. J. Eur. Math. Soc. (JEMS), 5(4):313-342, 2003.

[SCZ15] Laurent Saloff-Coste and Tianyi Zheng. Isoperimetric profiles and random walks on some permutation wreath products. To appear in Revista Matematica Iberoamericana, arXiv:1510.08830, 2015.

[SCZ16] Laurent Saloff-Coste and Tianyi Zheng. Random walks and isoperimetric profiles under moment conditions. Ann. Probab., 44(6):4133-4183, 2016. 
[SW90] Paolo M. Soardi and Wolfgang Woess. Amenability, unimodularity, and the spectral radius of random walks on infinite graphs. Math. Z., 205(3):471-486, 1990.

[Tes11] Romain Tessera. Asymptotic isoperimetry on groups and uniform embeddings into Banach spaces. Commentarii Mathematici Helvetici, 86(3):499-535, 2011.

[Tes13] Romain Tessera. Isoperimetric profile and random walks on locally compact solvable groups. Rev. Mat. Iberoam., 29(2):715-737, 2013.

YuVAl PeRes

One Microsoft Way, Redmond, WA 98052

E-mail address: peres@microsoft.com

TiAnYi ZHENG

Department of Mathematics, UC San Diego, 9500 Gilman Dr. La Jolla, CA 92093

E-mail address: tzheng2@math.ucsd.edu 